\title{
Mother knows best: Mothers are more egocentric towards their own child's bodily emotions
}

Louise P. Kirsch ${ }^{1}$, Michal Tanzer ${ }^{2}$, Maria Laura Filippetti ${ }^{3}$, Mariana von Mohr ${ }^{4}$, Aikaterini Fotopoulou $^{2}$

${ }^{1}$ Université de Paris, CNRS, Integrative Neuroscience and Cognition Center, F-75006 Paris, France

${ }^{2}$ Research Department of Clinical, Educational and Health Psychology, University College London, UK

${ }^{3}$ Centre for Brain Science, Department of Psychology, University of Essex, Colchester, UK

${ }^{4}$ Lab of Action and Body, Department of Psychology, Royal Holloway, University of London, United Kingdom

* Authors share first authorship

Corresponding authors:

Aikaterini Fotopoulou, Research Department of Clinical, Educational and Health Psychology, University College London, UK. E-mail: a.fotopoulou@ucl.ac.uk

Louise P. Kirsch, Université de Paris, CNRS, Integrative Neuroscience and Cognition Center, 45 rue des Saints Pères, 75006, Paris, France. E-mail: louise.kirsch@u-paris.fr 


\begin{abstract}
Our emotional state can influence how we understand other people's emotions, leading to biases in social understanding. Yet these biases have not been studied in specific relationships such as parent-child dyads, where not only understanding but also emotional and bodily regulation is key. We first conducted two experiments in adult stranger's dyads (total $\mathrm{N}=75$ ) to validate a novel, bodily Emotional Egocentricity Task (bEET) that allows concurrent affective, tactile stimulation in a dyad. We showed its effectiveness in eliciting emotional and bodily-emotional egocentric biases (EBs). We then recruited 68 mother-child dyads and found that mothers exhibit higher emotional and bodily EB towards their own than an unfamiliar child, but children showed only general, non-mother specific, egocentricity effects. Results suggest that mothers tend to use their bodily feelings more when judging the states of their own child than those of other children, possibly consistent with their regulatory parental role.
\end{abstract}

Keywords: emotional biases, egocentricity, altercentricity, caregiver-child dyad, embodied cognition

\title{
Statement of relevance
}

The ability to understand other's mind is influenced by our own emotional states leading to egocentric bias (EB) in how we interpret or perceive other's feelings. This basic human ability influences every-day social interactions, such as caregiver-child relations. However, how EB is shaped in a mother-child relation remains unknown, despite its societal and developmental importance. Focusing on mother-child dyads, we found that mothers tend to project their bodily feelings into their own child more than to other children. This projection may be part of the mechanisms that help caregivers regulate their children's feelings and 
bodily states. Relying more in own bodily states and feelings may be a way for parents to ensure the adaptive regulation of children to their physical and social environment.

\section{Introduction}

The ability to understand other's mental and affective states is a fundamental part of social communications. To understand other minds, we tend to partly rely on our own concurrent mental states that we project, or externalise to others (Mitchell, 2009), and vice versa, our own experiences may be influenced by how we read and 'internalise' others' states (Böckler et al., 2017; Dimaggio et al., 2008). Converging neuroimaging and neuromodulation evidence has supported and extended these behavioural findings, revealing shared neural representations between self and other, such as people acting and observing others act (Gazzola \& Keysers, 2009), thinking of themselves and others, or sharing other's feelings (Lamm et al., 2011, 2016; Singer et al., 2004). Although there are ongoing debates regarding the precise mechanisms of such self-other processing overlap (Heyes \& Catmur, 2021; Lamm \& Majdandžić, 2015), there is agreement that this overlap is sufficient for social understanding only when experiences align. To enable successful social understanding beyond aligned, concurrent experiences, a distinction has to be made between our own and other's mental states, termed the self-other distinction (S-OD; Brass et al., 2009; Lamm et al., 2016). Failure to make this distinction during incongruent experiences can lead to difficulties in inhibiting one's own mental states when judging the ones of others, the so-called egocentricity bias (EB; Gilovich et al., 1983; Steinbeis, 2016). Conversely, the disproportionate influence of other people's mental states on our own is known as altercentricity bias (AB; Hoffmann et al., 2016). The S-OD has been studied in sensorimotor and cognitive domains (Cunningham \& Turk, 2017), such as our ability to inhibit automatic imitation of actions (Brass et al., 2009; Santiesteban et al., 2012), or understand the mental 
states of others even when they conflict with our own (Lamm et al., 2016; Steinbeis, 2016). Moreover, these studies have converged on the finding that the S-OD relies on brain areas such as the temporo-parietal junction that is also required for mental inference and attribution (Decety \& Lamm, 2007). Interestingly, more anterior parts of this region and particularly the right supramarginal gyrus (Sebastian et al., 2012; Silani et al., 2013; Steinbeis, 2016) are implicated in the more affective dimension of the S-OD, such as when we need to inhibit our own emotions in order to empathise with another's experience (Lamm et al., 2016; Silani et al., 2013; Steinbeis, 2016).

Importantly, the ability for S-OD varies across the lifespan. Several studies have found that children, adolescents and older adults, compared to young and middle-aged adults, show increased EBs in cognitive and affective domains (Hoffmann et al., 2015; Riva et al., 2016, 2022; Steinbeis, 2016). Most existing studies on S-OD have however been conducted between participants that were previously unfamiliar with each other and therefore cannot account for important features of social understanding in established relationships. For example, social psychology studies on married couples have long shown that EBs may not be synonymous to reduced accuracy in social understanding (Gagné \& Lydon, 2004 for review). Instead, such biases may be caused by independent processes relating to the actual similarity and common goals between married couples, leading individuals, for example to project self and relationship ideals to their judgments of their partners. Indeed, such EBs have been associated with increased empathic understanding, closeness and satisfaction in married couples (Kenny \& Acitelli, 2001; Murray et al., 2002). It thus appears that in established relationships, egocentricity and other failures of the S-OD may confer long-term relational advantages.

Only questionnaire studies have assessed similar biases in parent-child relationships, finding that parents can be positively and egocentrically biased in how they evaluate their 
children (Lopez-Perez \& Wilson, 2015). Indeed the unique role of parents own emotions in the affective regulation of their children is central in existing developmental theories of affect regulation (Fonagy et al., 2019; Fotopoulou \& Tsakiris, 2017; Gergely \& Unoka, 2008). In early infancy, there are requirements for regulatory biobehavioural synchrony between infants and their parents (Feldman, 2007), with infants and caregivers engaged in sharing and co-regulating their embodied states (Fotopoulou et al., 2022). Over time, these repeated, synchronised interactions shape children's self and social understanding. Crucially, as children's cognition and selfhood matures, the parent-child dyad and its regulatory role also changes (Gergely \& Unoka, 2008; Fonagy et al., 2019; Fotopoulou et al., 2022). Parents do not just need to understand their children's current needs, but rather they need to teach their children anticipatory regulation strategies based on cultural and other shared, social or physical contextual factors (in physiological terms referred to as 'allostatic' regulation: Atzil et al., 2018; Fotopoulou, 2021; Fotopoulou \& Tsakiris, 2017; Theriault et al., 2021). For example, a father may need to teach their child to cover themselves with a blanket at night if he himself feels cold and hence anticipates, or predicts a cold night. Thus, as in the case of married couples, EBs may not signify failures of empathic understanding, but serve anticipatory regulation.

To capture this important facet in parent-child dyads, namely the predictive regulation of bodily feelings (Fotopoulou \& Tsakiris, 2017; Atzi et al., 2018; Fotopoulou et al., 2022), we developed and validated (Experiments 1a and 1b) a new bodily, Emotional Egocentricity Task (bEET), which we then tested in 68 child-mother dyads (Experiment 2). Dyads of participants are tested in proximal space using a set-up that allows tactile stimulation of participant's unseen arm at the same time as they are watching another participant's arm being touched, allowing a concurrent, unisensory experience of self (tactile only) and other (vision only). These experiences can either be congruent or incongruent in pleasantness 
(pleasant or unpleasant), and unlike previous studies that have used affective images during congruent stimulation, both participants are seeing the other being touched directly on the body, as they are feeling touch in the corresponding body part. We also tested unisensory (non-concurrent) perception for both the felt touch on the self and the seeing touch on the other. These task characteristics increase the relevance of the task in the social understanding and regulation of bodily feelings (Fotopoulou et al., 2022). Participants had to judge (Figure 1), how pleasant was the touch they felt (self) or saw (other), without knowing in advance which question they will have to answer, so they had to focus on both self and other's experience. This paradigm allowed the calculation of both $\mathrm{EB}$ and $\mathrm{AB}$ (based on congruency baselines as in previous studies), as well as new bodily, bEB and bAB measures (based on unisensory baseline measures; felt touch on the self and vicarious touch on the other). The latter are important for minimising the effect of differences in unisensory perception, which are particularly relevant here as mothers and children may have underlying age differences in unisensory perception. Moreover, these bodily measures can also control for familiarity, or salience effects in unisensory perception between familiar and unfamiliar dyads, e.g. the comparison between the egocentricity towards one's own child versus another child during congruent stimulation, is controlled by the unisensory (vicarious) perception of one's own versus the other child, respectively, beyond conditions of congruency as measured in the original task. We did not expect differences between the original and bodily measures, but we calculated both to provide grounds for comparison to the existing literature. Using the bEET, we expected children to show greater egocentricity than mothers overall, but we also expected differences on the S-OD for both mothers and children when tested within the familiar than the unfamiliar dyad. Specifically, we expected children to show greater AB and bAB towards their own mothers, given the salient, emotional role of the latter in children's life, but thus depending on their developmental stage (see Southgate, 2020 for the 
antecedents of this hypothesis in infancy). We also expected mothers to show greater emotional EB and bEB towards their own than unfamiliar children given the importance of their own emotional perspective in regulating their children.

\section{Methods}

All three experiments were conducted in accordance with the Declaration of Helsinki and were approved by the Ethics Committee of the Research Department of Clinical, Educational and Health Psychology, University College London. Written, informed consent was obtained from all participants prior to their participation.

\section{Experiment 1: Validation of the bodily Emotional Egocentricity Task (bEET)}

\section{Experiment 1a}

Participants. Forty-five participants (29 Females, 16 Males, $\mathrm{M}_{\mathrm{age}}=34.72, S D_{\text {age }}=10.68$, Range $_{\text {age }}=20-65$ years ; 37 right-handed, 4 left-handed and 2 ambidextrous) took part during a public event at the Royal Institution, London.

\section{Materials and procedure}

Touch stimuli. Touch was applied on a $9 \mathrm{~cm}$ long area on the participant's forearm. Participants' forearm was marked beforehand to ensure the touch would be delivered in the same location for both participants. Tactile stimuli consisted of two different balls of material (cotton ball and scourer). Touch was applied by a trained experimenter at constant pressure and speed simultaneously on the two participants' forearms, lasting for 3 seconds and repeated twice per trial. This $3 \mathrm{~cm} / \mathrm{s}$ speed was chosen as known to be optimal for CT fibres stimulation (Morrison et al., 2010). For details on the experimental set-up see supplementary materials. 


\section{Bodily emotional egocentricity bias in mother-child dyads}

Procedure. Participants first filled questionnaires assessing empathy (Interindividual Reactivity Index; IRI, Davis, 1983) and autism traits (short autism questionnaire 10; AQ10; Allison et al., 2012), and then were paired with an unfamiliar, same gender, and similar age (when possible) participant (Figure 1). Due to experimental administration error the selfreport questionnaires for three participants are missing. A curtain was separating participants, to avoid any view of the 'other'. The experimenter explained the task and gave both participants a booklet where they circle with a pen their ratings on a scale from 0 'not at all pleasant' to 10 'very pleasant'. Participant on the right of the experimenter was asked to place their right forearm in the box in front of them; whereas the participant on the left was asked to place their left forearm in the box. Participants could not see their own forearm and were instructed to always look at the 'other' participant's forearm and focus on both the pleasantness of the touch they felt, and the pleasantness for the other of the touch they saw. Participants did not know before the end of the touch which of the two questions they would be asked after the touch: i.e. either "How pleasant was the touch for YOU?" or "How pleasant was the touch for the OTHER?". Each pair of participants received simultaneous tactile stimulation with either identical (Congruent condition) or different (Incongruent condition) materials. The experimenter carried out two practice trials, and then 2 blocks of 8 trials, with a short break between the two blocs. All trials were pseudo-randomized. 


\section{A. Design Main Task}

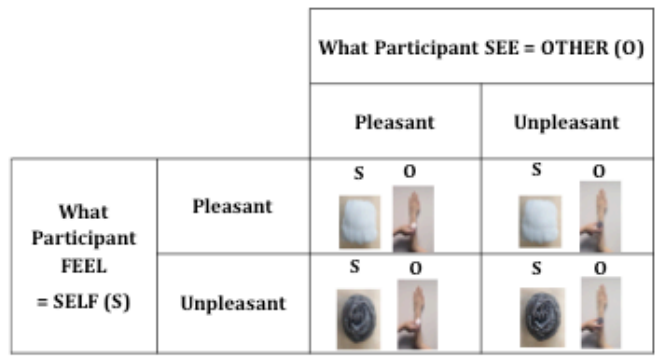

B. Procedure

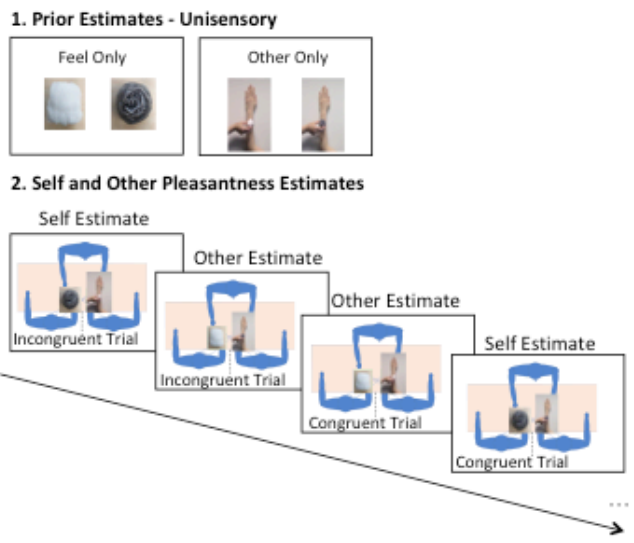

\section{Set-up Experiment 1}

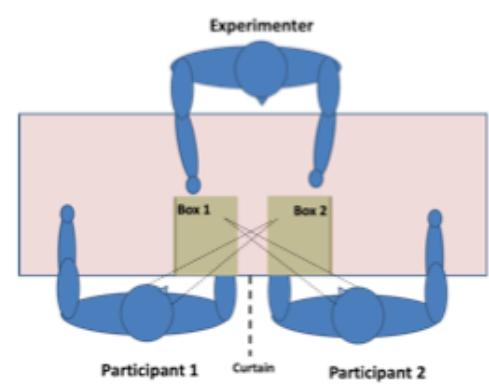

D. Set- up Experiment 2
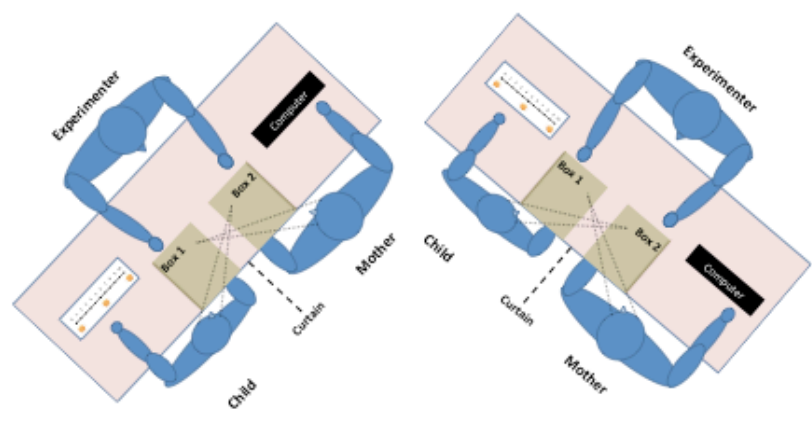

Figure 1. A. Experimental Design. Valence of the fabric touching the self and the other being either pleasant/unpleasant and congruent/incongruent. B. Procedure. C. Experimental Set-up for Experiment $1 a$ and $1 b$. D. Experiment 2 experimental set up, enabling two Mother-Child dyads to receive separate but simultaneous tactile stimulation to their concealed forearm whilst observing tactile stimulation upon their partner. Note that in all experiments participants could not see their own forearm but only the other participant's forearm (box closed on the top but open on the other participant's side).

\section{Experiment 1b - 'Replication' of the bEET}

Participants. Thirty participants took part in Experiment $1 \mathrm{~b}$ and were recruited via an online SONA system (18 Females, 12 Males, $\mathrm{M}_{\mathrm{age}}=25.00, \mathrm{SD}_{\mathrm{age}}=7.91$, Range $\mathrm{age}_{\mathrm{ag}}=19-56$; years, $S D=7.91$ years; all right-handed). All participants were compensated for their time.

Procedure. Experiment $1 \mathrm{~b}$ followed the same procedure used as in Experiment $1 \mathrm{a}$ in a laboratory-controlled setting, with a few changes. First, a pair of materials was added (synthetic wool as pleasant material and velcro as unpleasant) to increase variability and to increase power, leading to a total 32 trials in the main task. Incongruent pairs were kept constant and always had opposite valence (one pleasant and the other unpleasant). In 
Experiment $1 \mathrm{~b}$ participants made all their ratings on a computer, using a continuous analogue scale from 0 to 100,0 being 'not at all pleasant' and 100 being 'extremely pleasant', requiring data entry via the trackpad for collection in MATLAB. Moreover, two extra measures were added as baselines (before the main task): Touch only condition: where participants had to rate how pleasant was the touch they felt (pleasantness rating of their own tactile experience), with no vision of self nor other, and a Vision only condition: where participants had to rate the pleasantness of all the materials with no one being touched (twice for each object and each condition).

\section{Experiment 2. Bodily Emotional Egocentricity in Mothers and Children}

Participants. Hundred thirty-six participants took part in Experiment 2 during two public events ("FunFamily Day" at the Royal Institution, London; and "Self Impressions" held at the TATE Modern). Participants were recruited in pairs of mother and child (Mothers: $n=68$, $\mathrm{M}_{\mathrm{age}}=43.1, \mathrm{SD}_{\mathrm{age}}=4.9$, Range $_{\mathrm{age}}=30-57$ years; Children: $\mathrm{n}=68,22$ boys and 43 girls, $\mathrm{M}_{\mathrm{age}}=8.3$, $\mathrm{SD}_{\text {age }}=2.3$, Range $\mathrm{age}=5-15$ years), to be tested either separately or together, but always simultaneously by two experimenters. Given developmental findings on children's ability to take another person perspective not to develop before the age of seven 1956) and that other studies used seven years old as a standard cut-off (Riva et al., 2016; Steinbeis, 2016), children that were younger than seven were excluded from further analysis $(n=16)$. Moreover, an additional 7 children did not complete the full task, and were discarded (final children sample: $\mathrm{n}=45,31$ girls, $\mathrm{M}_{\mathrm{age}}=9.24, \mathrm{SD}_{\mathrm{age}}=2.1$, Range $_{\mathrm{age}}=7-15$ years).

Task and procedure. Experiment 2 followed the same design and procedure as Experiment 1a, with three exceptions: (i) Experiment 2 was conducted as a between-subject design, with mothers and children paired with either their own child $(n=35) /$ mother $(n=22)$ or other child $(n=33) /$ mother ( $n=23)$ (Figure 1.D). (ii) Children were asked to point to the relevant number 
in front of them on a paper-based scale, which was illustrated with cartoon faces to denote negative (unhappy) and positive (happy) affect, ranging from $0=$ not at all pleasant/unhappy to $10=$ extremely pleasant/happy; whereas for the mothers, the pleasantness scale was presented on a computer ( 0 to 100 VAS scale), as in Experiment $1 \mathrm{~b}$. In order to compare between the two groups children's ratings were rescaled. (iii) as in Experiment 1b, two extra measures were also added as baselines: Touch only condition and Vision only condition. As in Experiment 1, mothers answered demographics questions as well as the IRI (Davis, 1983). Children answered the equivalent questionnaire developed for children (EmQue-CA, Overgaauw et al., 2017) measuring both affective and cognitive empathy as well as prosocial motivation. Due to technical error, only 28 children answered this questionnaire. Each experiment lasted for about 20 minutes.

\section{Statistical analysis}

We first computed the AB and the EB. Similarly to previous studies (Hoffmann et al., 2016; Silani et al., 2013), EB was computed as the average of the difference between incongruent and congruent trials when participants had to judge the pleasantness of the touch for the other participant and $\mathrm{AB}$ was computed similarly but in trials when participants had to judge the pleasantness of the touch they felt.

$\mathrm{EB}=\frac{\text { (Incongruent Other Unpleasant-Congruent Other Unpleasant })+(-1 *(\text { Incongruent Other Pleasant-Congruent Other Pleasant }))}{2}$ $\mathrm{AB}=\frac{((\text { Incongruent Self Unpleasant }- \text { Congruent Self Unpleasant })+(-1 *(\text { Incongruent Self Pleasant-Congruent Self Pleasant }))}{2}$

Note that the valence label corresponds to what the target was feeling, e.g. 'Incongruent Other Unpleasant" corresponds to a condition where the 'self' was feeling a pleasant touch while seeing the 'other' being touched with an unpleasant material.

To confirm the existence of these bodily-emotional biases, in line with previous studies (Hoffmann et al., 2016; Silani et al., 2013), we computed an EEB score (EB-AB) and 
used one sample t-tests to test whether EEB, AB and EB were significantly different from zero.

To test our hypothesis regarding the size of the biases (AB and EB) multilevel models (MLM) were used. Bias score (as computed from $\mathrm{AB}$ and $\mathrm{EB}$ score) was served as continuous dependent variables, with bias type (AB vs. EB) and age as fixed factors and participants as a random effect. For Experiment 2, statistical analysis was similar to Experiment 1, except that pairs (Own vs. Other) was also added as a fixed between variable.

Next, in Experiment 1b and Experiment 2, using the baseline measures, we computed a new measure of bodily $\mathrm{EB}$ and $\mathrm{AB}$ (bAB and $\mathrm{bEB}$ ). These were averaged across valences (Pleasant and Unpleasant). Each bodily-emotional bias was computed as follow:

$\mathrm{bEB}=$

$\underline{\text { |Other Incongruent Unpleasant - Only Vision Unpleasant) }|+| \text { (Other Incongruent Pleasant - Only Vision Pleasant)| }}$

$\mathrm{bAB}=$

$\underline{\text { |Self Incongruent Unpleasant - Only Tactile Unpleasant)|+|(Self Incongruent Pleasant - Only Tactile Pleasant)| }}$

Separate linear models were used in order to test our hypothesis regarding the size of the bodily biases. For Experiment 2 the same data analyses were conducted, but again pairs (Own vs. Other) was added as a fixed variable. When applicable, Bayesian statistics were performed using JASP in order to calculate Bayes Factors $\left(\mathrm{BF}_{10}\right)$ and to allow further interpretation of the observed effects, in particular, the extent to which data provided support for the alternative versus null hypotheses.

To explore the relationships between the biases (AB, EB, EEB) and social cognition measures (i.e., AQ10, and IRI) we combined the data from Experiment 1a and Experiment 1b and run an exploratory Spearmen partial correlation analysis, controlling for age and experiment (i.e., Experiment 1 or Experiment 1b). For the mother and the children group we also explored the relationships of $\mathrm{bEB}$ and $\mathrm{bAB}$ with their social cognition measures (as 
measured by the AQ and IRI in mothers and EmQu-CA in children); however, results for these correlations should be considered with caution given the limited subsample.

\section{Results}

\section{Experiment 1: Validation of the bodily Emotional Egocentricity Task (bEET)}

\section{Experiment 1a.}

Emotional $\boldsymbol{E B}$ and $\boldsymbol{A B}$. One sample t-tests showed that $\mathrm{EB}, \mathrm{AB}$ and $\mathrm{EEB}$ were significantly different from zero $\left(\mathrm{EB} ; t_{(44)}=4.91, p<0.001, \mathrm{~d}=.73, \mathrm{BF}^{10}=1564.5 ; \mathrm{AB} ; t_{(44)}=3.07, p=0.004\right.$, $\left.\mathrm{d}=.46, \mathrm{BF}_{10}=9.27 ; \mathrm{EEB} ; t_{(44)}=2.43, p=0.02, \mathrm{~d}=.36, \mathrm{BF}_{10}=2.28\right)$. Moreover, using MLM a main effect of bias type (EB vs. AB) emerged ( $b=4.44, S E=1.82, p=0.02$; Figure 2.A), indicating a significant difference between $\mathrm{EB}$ and $\mathrm{AB}$, while controlling for age $\left(\mathrm{M}_{\mathrm{EB}}=9.44, S E=1.92\right.$ $\left.95 \% C I[5.6513 .02] ; \mathrm{M}_{\mathrm{AB}}=5.00, S E=1.63,95 \% C I[1.218 .57]\right)$. 
A. Experiment 1a-Emotional Biases

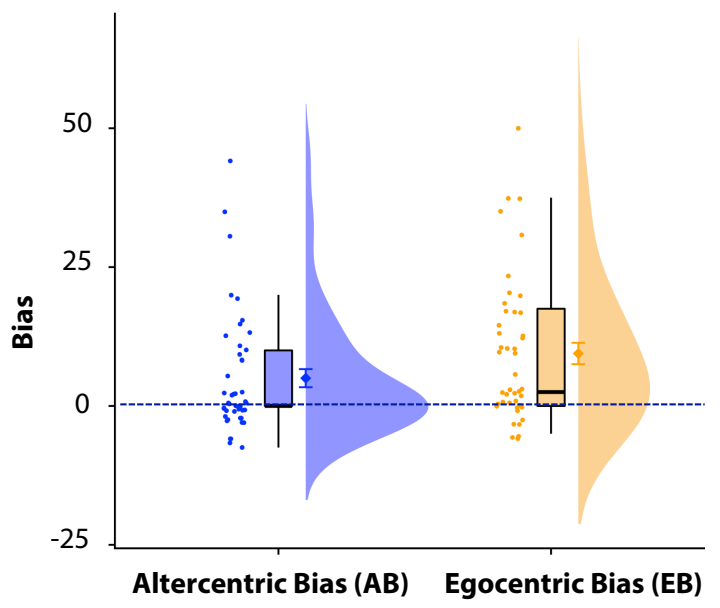

B. Experiment $1 \mathrm{~b}-$ Emotional Biases

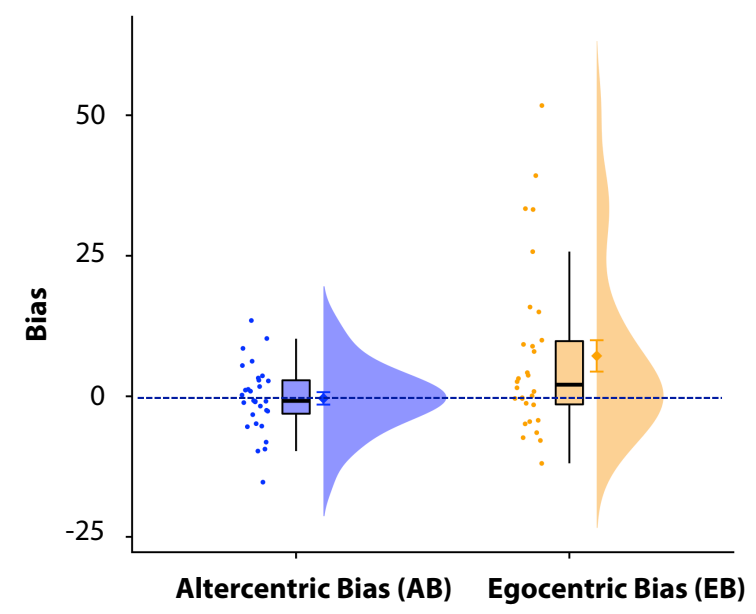

C. Experiment $1 \mathrm{~b}-$ Bodily Emotional Biases

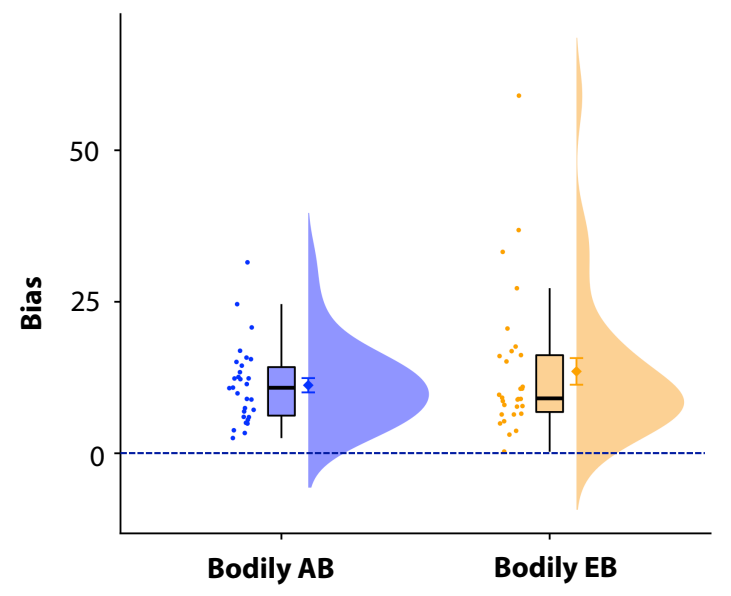

Figure 2. Egocentric and Altercentric Biases $(E B / A B)$ in Experiment 1. A. In Experiment 1a. B. In Experiment 1b. C. Bodily altercentric (bAB) and egocentric (bEB) biases found in Experiment $1 b$. Jittered dots represent biases for each individual participant. Boxplot presents the central tendency. The half violin provides data distribution. Diamond represent the mean for each bias, and the error bars denotes the standard error of the mean.

\section{Experiment 1b}

Emotional $\boldsymbol{E B}$ and $\boldsymbol{A B}$. One sample t-test showed that while EB and EEB were significantly different from zero $\left(\mathrm{EB}: t_{(29)}=2.57, p=0.01\right.$, Cohen's $\mathrm{d}=0.47, \mathrm{BF}_{10}=3.13$; EEB: $t_{(29)}=3.07$, $p=0.005$, Cohen's $\left.\mathrm{d}=0.56, \mathrm{BF}_{10}=8.72\right), \mathrm{AB}$ was not $\left(t_{(29)}=-0.33, p=0.74\right.$, Cohen's $\mathrm{d}=-0.06$, $\mathrm{BF}_{10}=0.205$ ). In addition, using MLM a main effect of bias type (EB vs. AB) emerged indicating a significant difference between $\mathrm{EB}$ and $\mathrm{AB}$, while controlling for age $(b=7.78$, 
$S E=2.53, p=0.004 ; \mathrm{M}_{\mathrm{EB}}=7.57, S E=2.3595 \% C I[1.77-13.38] ; \mathrm{M}_{\mathrm{AB}}=-0.19, S E=2.35,95 \% C I[-$ 6.00 5.61]; Figure 2.B).

$\boldsymbol{b E B}$ and $\boldsymbol{b} A \boldsymbol{B}$ effects. After confirming the existence of emotional $\mathrm{EB}$ in our newly developed bEET, we investigated the new bodily biases. One sample t-tests showed that both bEB $\left(t_{(29)}=6.07, p<0.001\right.$, Cohen's $\left.\mathrm{d}=1.12, \mathrm{BF}_{10}=5.2 \mathrm{e}+7\right)$ and $\mathrm{bAB}\left(t_{(29)}=9.51, \mathrm{p}<0.001\right.$, Cohen's $\mathrm{d}=1.74, \mathrm{BF}_{10}=1.7 \mathrm{e}+4$ ) were significantly different from zero, confirming the existence of these bodily-emotional biases (Figure 2.C).

Correlations with social cognition measures. Partial correlation analysis yielded a negative correlation between autistic trait (as measured by the AQ10) and the $\operatorname{EEB~}\left(r_{(71)}=-.31, p=.008\right.$, $p_{\text {FDRcorrected }}=0.06$ ), suggesting that those who experienced larger EEB have lower autistic traits. In addition, a positive correlation between the AB and IRI fantasy scale emerged $\left(r_{(71)}=.37, p=.002, p_{\text {FDRcorrected }}=0.03\right)$, suggesting that those who show higher AB also report higher tendency to transpose themselves imaginatively into the feelings and actions of fictious characters.

\section{Experiment 2: Emotional biases in Mothers and Children}

Emotional $\boldsymbol{E B}$ and $\boldsymbol{A B}$ in mothers. We found a two-way interaction between bias type (EB vs $\mathrm{AB}$ ) and being paired with own vs. other child ( $b=11.49, S E=4.63, p=.02$; Figure 3.A). Probing this interaction using planned comparisons, revealed that this effect was driven from a larger EB in the group that was paired with their own child as compared to the group that was paired with a stranger (i.e., other child) $(t=3.40, p<.001,95 \% C I[4.96 ; 18.75]$; $\left.\mathrm{M}_{\mathrm{EB} \_ \text {own }}=16.36, S E=3.54 ; \mathrm{M}_{\mathrm{EB} \_ \text {Other }}=4.77, S E=2.22 ; \mathrm{BF}_{10}=4.78\right]$. There was no difference in $\mathrm{AB}$ between the two groups $\left(t=0.10, p=.92,95 \% C I[-6.53 ; 7.26] ; \mathrm{M}_{\mathrm{AB} \_ \text {own }}=3.32, S E=2.44\right.$; $\left.\mathrm{M}_{\mathrm{AB} \_ \text {Other }}=3.00, S E=2.55 ; \mathrm{BF}_{10}=0.25\right)$. In addition, the difference between $\mathrm{EB}$ and $\mathrm{AB}$ was significant only within the group that was paired with their own child (own child: $t=4.08$, 
$p<.001,95 \% C I[6.72 ; 19.61], \mathrm{BF}_{10}=26.07$; other child $t=0.50, p=.62,95 \% C I[-4.92 ; 8.31]$

$\left.\mathrm{BF}_{10}=0.22\right)$.

A. Emotional Biases in Mothers

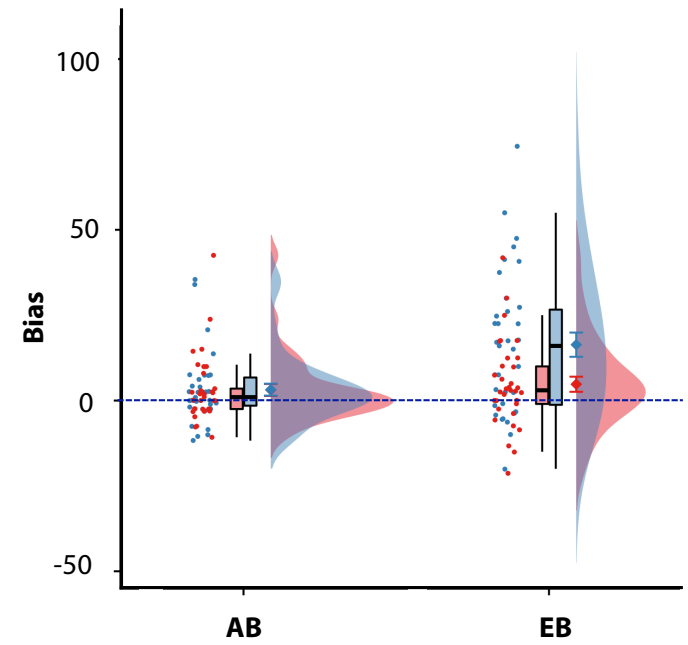

C. Emotional Biases in Children

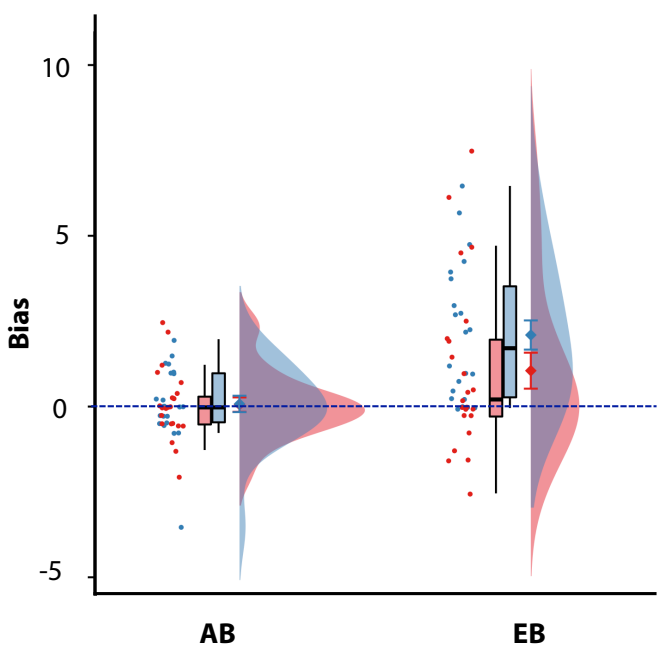

B. Bodily Emotional Biases in Mothers

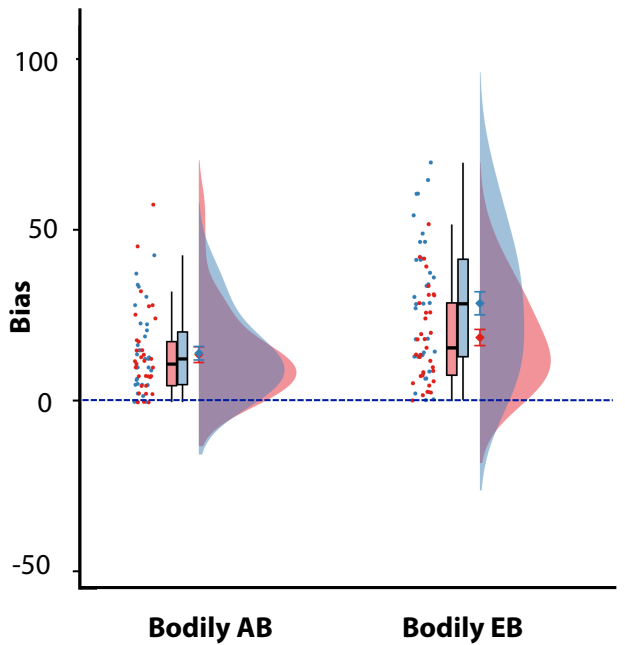

D. Bodily Emotional Biases in Children

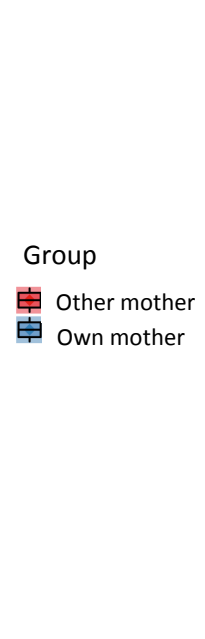

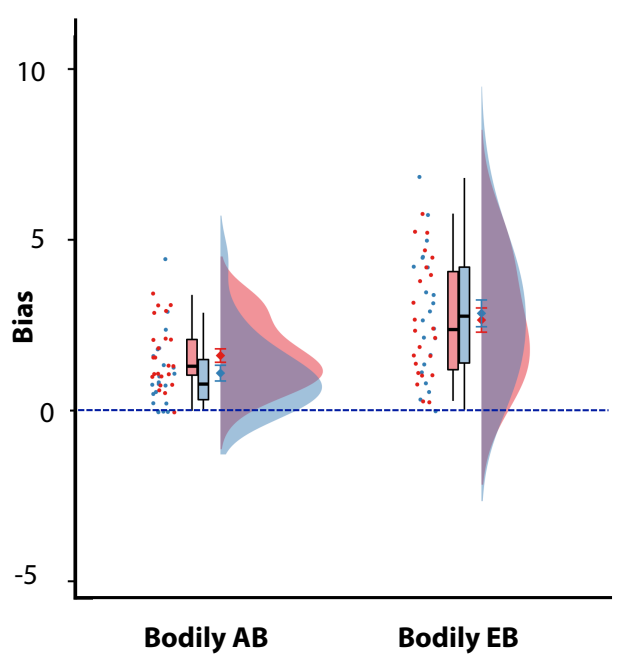

Figure 3. Emotional biases in mothers. A. Emotional Egocentric (EB) and Altercentric Biases (AB) in Experiment 2 in mothers paired with their own or other child. B. Bodily Emotional Egocentric (bodily EB) and Altercentric Biases (bodily AB) in Experiment 2 in mothers paired with their own or other child. C. Emotional Egocentric (EB) and Altercentric Biases (AB) in Experiment 2 in children paired with their own or other mother. D. Bodily Emotional Egocentric (bodily EB) and Altercentric Biases (bodily AB) in Experiment 2 in children paired with their own or other mother. Jittered dots represent biases for each individual participant. Boxplot presents the central tendency. The half violin provides data distribution. Diamond represent the mean for each bias, and the error bars denotes the standard error of the mean. 
$\boldsymbol{b E B}$ and $\boldsymbol{b} A B$ effects in mothers. First, one sample t-tests showed that both bEB (Own: $t_{(33)}=8.32, p<0.001$, Cohen's d=1.42, $\mathrm{BF}_{10}=8.91 \mathrm{e}+6$; Other: $t_{(32)}=2.03 \mathrm{e}+6, p<0.001$, Cohen's $\left.\mathrm{d}=1.36, \mathrm{BF}_{10}=2.03 \mathrm{e}+6\right)$ and $\mathrm{bAB}\left(\mathrm{Own}_{\mathrm{B}} \mathrm{t}_{(33)}=7.20, \mathrm{p}<0.001\right.$, Cohen's $\mathrm{d}=1.23, \mathrm{BF}_{10}=4.71 \mathrm{e}+4$; Other: $t_{(32)}=6.05, p<0.001, \mathrm{~d}=1.05, \mathrm{BF}_{10}=1.88+3$ ) were different from zero (Figure 2.C). In addition, for bEB a significant main effect of pairs (Own Vs. Other) was found, indicating that the group that was paired with their own child showed a larger bEB $(b=-9.23, S E=3.99$, $\left.p=0.02,95 \% C I[-17.21 ; ;-1.24], \mathrm{BF}_{10}=1.88\right)$, but not for $\mathrm{bAB}(b=-0.24, S E=2.99, p=0.93$, $95 \% C I[-5.73 ; 6.21] \mathrm{BF}_{10}=0.25 ;$ Figure 4.B).

Emotional $\boldsymbol{E B}$ and $\boldsymbol{A B}$ in children. A main effect of bias type was found $(b=-1.51, S E=0.37$, $t=-4.01, p<0.001 ; \mathrm{M}_{\mathrm{EB}}=1.61, S E=0.2695 \% C I[1.00 ; 2.21] ; \mathrm{M}_{\mathrm{AB}}=0.09, S E=0.0995 \% C I[-$ $0.51 ; 0.70]$ Figure 3.C) indicating a larger EB relative to AB. However, unlike the mother group, we did not find a significant two-way interaction between target and being paired with own vs other child ( $b=1.02, S E=0.75, t=1.36, p=0.17)$.

$\boldsymbol{b E B}$ and $\boldsymbol{b} \boldsymbol{A B}$ in children. First, similar to the mother group one sample t-tests showed that both bEB (Own: $t_{(21)}=7.21, p<0.001$, Cohens' $\mathrm{d}=1.53, \mathrm{BF}_{10}=4.02 \mathrm{e}+4$; Other: $t_{(22)}=7.41$, $p<0.001, \quad$ Cohens'd $\left.=1.54, \quad \mathrm{BF}_{10}=7.73 \mathrm{e}+4\right) \quad$ and $\quad \mathrm{bAB} \quad\left(\mathrm{Own}: \quad \mathrm{t}_{(22)}=4.79, \quad p<0.001\right.$, Cohens'd=1.02, $\mathrm{BF}_{10}=277.89$; Other: $t_{(22)}=8.22, p<0.001$, Cohens'd=1.71, $\mathrm{BF}_{10}=3.74 \mathrm{e}+5$ ) were different from zero. In addition, for $b E B$, we did not find a significant main effect of pairs (Own Vs. Other) $\left(b=-0.22, S E=0.51, p=.66,95 \% \mathrm{CI}[-1.25 ; 0.81], \mathrm{BF}_{10}=0.31\right)$, however for $\mathrm{bAB}$ there is a trend towards an effect of pairs $(b=0.55, S E=0.28, p=.06,95 \% C I[-$ $\left.0.01 ; 1.12], \mathrm{BF}_{10}=0.93\right)$, indicating a larger $\mathrm{bAB}$ for children that were paired with another mother as compared to their own. Note that this effect was not confirmed by the BF, so no conclusion can be drawn on that comparison $\left(\mathrm{BF}_{10}<1\right)$ (Figure 2.C).

Comparing EEB effect between mothers and children groups. First, we confirmed that in both groups EEB was different from zero (Mothers: $\mathrm{M}_{\mathrm{EEB}}=7.59 t_{(67)}=3.15, p<0.001$, $\mathrm{d}=0.38$, 
$\mathrm{BF}_{10}=11.81$; Children: $\left.\mathrm{M}_{\mathrm{EEB}}=15.01 t_{(44)}=3.81, p<0.001, \mathrm{~d}=0.57, \mathrm{BF}_{10}=64.75\right)$. Next, we compared between EEBs in both groups using linear models. A trend towards a larger EEB in the children group as compared to the mothers was found but was not confirmed by the BF, so no conclusion can be drawn on that comparison $(b=7.52, \mathrm{SE}=4.25, p=0.07,95 \% C I[-$ $\left.0.63 ; 16.23, \mathrm{BF}_{10}=0.76\right)$. Separate comparison for EEB in each condition (Other or Own) were also conducted, but there were no significant effects (see supplementary). Also, there was no significant difference between the bodily biases (see supplementary).

\section{Correlations with social cognition measures.}

Mother group. Partial correlation analysis yielded positive correlations between IRI empathy concern subscale and $\mathrm{EB}$ and $\operatorname{EEB}\left(r_{(63)}=.33, p=.008, p_{F D R c o r r e c t e d}=.053 ; r_{(63)}=.27 p=.03\right.$, $p_{F D \text { Rorrected }}=14$, respectively), suggesting that those who show higher EB also report more feelings of sympathy and concern for unfortune others. In addition positive correlations between $\mathrm{bAB}$ and IRI fantasy (i.e., the tendency to transpose oneself imaginatively into the feelings and actions of fictitious characters) and personal distress (i.e., self-oriented feelings of distress in interpersonal settings) subscales were found $\left(r_{(62)=.34,} p=.006\right.$, $p_{\text {FDRcorrected }}=0.053 ; r_{(62)}=.38, p=.002, p_{F D \text { corrected }}=0.04$, respectively). No other effects emerged.

Children group. Partial correlation analysis yielded positive correlations between $\mathrm{AB}$ and affective empathy $\left(r_{(20)}=.74, p<.001, p_{F D \text { Rorrected }}=0.003\right)$, suggesting that those who show higher $\mathrm{AB}$ scored higher on the affective empathy subscale and report more prosocial motivation $\left(r_{(20)}=.63, p=.003, p_{F D R c o r r e c t e d}=0.02\right)$. No other effects emerged.

\section{Discussion}

The ability to understand other minds relies on our own concurrent mental states and vice versa. Thus, to enable successful social and self-understanding beyond aligned, concurrent 
experiences, we have to be able to distinguish between our own and another's mental states, termed the S-OD. An important omission in the literature is the lack of studies on the S-OD in the context of key relational dyads, such as parent-child dyads. This is important as in everyday social interactions with close others our social understanding relies on both current and past relational experiences, representations and goals (Decety \& Jackson, 2006; Fonagy et al., 2019; Fotopoulou \& Tsakiris, 2017; Lamm et al., 2016; Trilla et al., 2021).

In the present study, given the key role of bodily feelings in parent-child dyads and the potential, sensory confounds of previous S-OD tasks in such dyads, we first successfully validated a new, bodily emotional S-OD task using two separate experiments with healthy adult volunteers. We confirmed the expected emotional egocentricity bias observed in previous studies (see Introduction), and were also able to calculate a bEB in concurrently tested dyads.

Using this task, we found evidence supporting our main hypothesis that mothers show greater emotional EB towards their own child than an unfamiliar child. Importantly, this was the case for both classical EB and the sensory-controlled, bEB measure, suggesting also a bodily emotional egocentric effect. A larger maternal EB towards their own child could be interpreted as a 'failure' of the S-OD, with mothers being possibly less attuned to the child's states than one's own. However, previous literature in social and development psychology suggests that this conclusion may not apply to established intimate relationships where egocentricity may have some relational advantages (see Introduction). Indeed, in our sample, higher EB in the mother group was associated with feelings of sympathy and concern for unfortune others in a self-report measure. Moreover, the larger, child-specific maternal EB and $\mathrm{bEB}$ we observed is consistent with previous questionnaire studies on parental egocentricity (Lopez-Perez \& Wilson, 2015). It is possible that the observed, child-specific maternal EB may be an indication of the requirements of anticipatory bodily and affective 
regulation (see Introduction). Parenting requires attending and responding not only to their children 'here and now' embodied and emotional needs (homeostastic regulation, Fotopoulou \& Tsakiris, 2017), but also anticipating future, counterfactual changes (Atzil et al., 2018; Fotopoulou et al., 2022; Theriault et al., 2021), that can be predicted by reading one's own embodied incongruent states. For example, parents may use their own body temperature to decide how best to dress their children for school. Or a parent may expect a child to try new tastes and eat their offered dinner in a special family occasion due to the enjoyment and sense of exploration parent themselves experiences in the given context. Thus, this novel finding suggest the S-OD has an adaptive and changing role in parenting and opens new avenues for research in the relationship between the S-OD and affective regulation.

As regards to our second hypothesis, we did not find conclusive evidence that children show greater $\mathrm{AB}$ towards their own than an unfamiliar mother, possibly due to the increased egocentricity of mothers towards their own child, or perhaps the relatively wide age range of our sample. These findings suggest that children's egocentricity may be a generic, non-parent oriented mechanism, and hence possibly not influenced by relational closeness. Future studies could test this hypothesis focusing on different age groups. However, despite the age range of our sample it should be noted that similarly to previous findings indicating that children were more egocentric than adults (Riva et al., 2016; Sebastian et al., 2012), we found a larger emotional $\mathrm{EB}$ relative to $\mathrm{AB}$ in children that was not related to the grouppairing condition.

Limitation of this study should be acknowledged. Firstly, even though we validated the bEET in three different experiments, our core examination of mother-child dyad was only tested once, calling for replication in future studies. Secondly, the mother-child experiment was conducted in a public event, which limited our ability to add more measures such as socioeconomic or educational status, attachment style or other developmental traits or state 
measures that were already shown to affect S-OD bias (Bukowski et al., 2020; Hoffmann et al., 2016) and potentially could have interacted with or accounted for the observed effects. Future studies should test this possibility and test this in a laboratory-controlled setting.

In conclusion, the present study offers novel insights on the nature of the self-other distinction in mother-child relationships. Using a novel paradigm designed to test emotional S-OD biases while controlling for sensory, salience and familiarity confounds, we report for the first time that mothers show greater emotional egocentricity towards their own child than another child, possibly due to the requirements of parental affective regulation and related relational goals.

\section{Open Practices Statement}

Neither of the experiments reported in this article was formally preregistered. De-identified data for all experiments are available on the Open Science Framework and on request to the authors (https://osf.io/xwvhg/?view_only=ce194464a86e4074aba47da43d15bf72)

\section{Acknowledgements}

This project was supported by the European Union's Horizon 2020 research and innovation programme under grant agreement No.818070, for the Consolidator Award METABODY (to AF). Thank you to all KatLab members who helped with this study, especially during the public events, to name a few, Laura Crucianelli, Sonia Ponzo, Emma Johnsson, Elena Panagiotopoulou. We are grateful to all the participants and families who to took part in the studies.

\section{Author Contributions Statement}

AF and LPK designed the study. AF, LPK, MLF and MM conducted testing and data collection. LPK and MT analysed the data. AF, LPK and MT wrote the manuscript. All authors approved the final version of the manuscript. 


\section{References}

Allison, C., Auyeung, B., \& Baron-Cohen, S. (2012). Toward brief "Red Flags" for autism screening: The Short Autism Spectrum Quotient and the Short Quantitative Checklist for Autism in toddlers in 1,000 cases and 3,000 controls [corrected]. Journal of the American Academy of Child and Adolescent Psychiatry, 51(2), 202-212.e7. https://doi.org/10.1016/j.jaac.2011.11.003

Atzil, S., Gao, W., Fradkin, I., \& Barrett, L. F. (2018). Growing a social brain. Nature Human Behaviour, 2(9), 624-636. https://doi.org/10.1038/s41562-018-0384-6

Böckler, A., Herrmann, L., Trautwein, F.-M., Holmes, T., \& Singer, T. (2017). Know Thy Selves: Learning to Understand Oneself Increases the Ability to Understand Others. Journal of Cognitive Enhancement, 1(2), 197-209. https://doi.org/10.1007/s41465-0170023-6

Brass, M., Ruby, P., \& Spengler, S. (2009). Inhibition of imitative behaviour and social cognition. Philosophical Transactions of the Royal Society B: Biological Sciences, 364(1528), 2359-2367. https://doi.org/10.1098/rstb.2009.0066

Bukowski, H., Boch, M., Lamm, C., \& Silani, G. (2020). Is Self-Other distinction malleable? Egocentric and altercentric biases in empathy are modulated by priming attachment style and similarity mindsets. PsyArXiv. https://doi.org/10.31234/osf.io/bpyvz

Cunningham, S. J., \& Turk, D. J. (2017). Editorial: A Review of Self-Processing Biases in Cognition. Quarterly Journal of Experimental Psychology, 70(6), 987-995. https://doi.org/10.1080/17470218.2016.1276609

Davis, M. H. (1983). Measuring individual differences in empathy: Evidence for a multidimensional approach. Journal of Personality and Social Psychology, 44(1), 113126. https://doi.org/10.1037/0022-3514.44.1.113 
Decety, J., \& Jackson, P. L. (2006). A social-neuroscience perspective on empathy. Current Directions in Psychological Science, 15(2), 54-58. https://doi.org/10.1111/j.09637214.2006.00406.x

Decety, J., \& Lamm, C. (2007). The role of the right temporoparietal junction in social interaction: How low-level computational processes contribute to meta-cognition. The Neuroscientist, 13(6), 580-593. doi: 10.1177/1073858407304654.

Dimaggio, G., Lysaker, P., H., Carcione, A., Nicolo, G., \& Semerari, A. (2008). Know yourself and you shall know the other... to a certain extent: Multiple paths of influence of self-reflection on mindreading-ScienceDirect. Consciousness and Cognition, 17(3), 778-789. https://doi.org/10.1016/j.concog.2008.02.005

Feldman, R. (2007). Parent-infant synchrony and the construction of shared timing; physiological precursors, developmental outcomes, and risk conditions. Journal of Child Psychology and Psychiatry, 48(3-4), 329-354. https://doi.org/10.1111/j.14697610.2006.01701.x

Fonagy, P., Gergely, G., Jurist, E. L., \& Target, M. (2019). Affect Regulation, Mentalization, and the Development of the Self. Routledge. https://doi.org/10.4324/9780429471643

Fotopoulou, A. (2021). Mentalising allostasis: The sense that I should eat: Comment on 'The sense of should: A biologically-based framework for modeling social pressure' by Jordan E. Theriault, Liane Young, and Lisa Feldman Barrett. Physics of Life Reviews, 36, 20-23. https://doi.org/10.1016/j.plrev.2020.09.002

Fotopoulou, A., \& Tsakiris, M. (2017). Mentalizing homeostasis: The social origins of interoceptive inference. Neuropsychoanalysis, 19(1), 3-28. doi: $10.1080 / 15294145.2017 .1294031$ 
Fotopoulou, A., von Mohr, M., \& Krahé, C. (2022). Affective regulation through touch: Homeostatic and allostatic mechanisms. Current Opinion in Behavioral Sciences, 43, 80-87. doi: 10.1016/j.cobeha.2021.08.008

Gagné, F., M., \& Lydon, J., E. (2004). Bias and Accuracy in Close Relationships: An Integrative Review. Personality and Social Psychology Reviews, 8(4), 322-338. https://doi.org/10.1207/s15327957pspr0804_1

Gazzola, V., \& Keysers, C. (2009). The observation and execution of actions share motor and somatosensory voxels in all tested subjects: Single-subject analyses of unsmoothed fMRI data. Cerebral Cortex, 19(6), 1239-1255. doi: 10.1093/cercor/bhn181

Gergely, G., \& Unoka, Z. (2008). Attachment and mentalization in humans: The development of the affective self. In E. L. Jurist, A. Slade, \& S. Bergner (Eds.), Mind to mind: Infant research, neuroscience, and psychoanalysis (pp. 50-87).

Gilovich, T., Jennings, D. L., \& Jennings, S. (1983). Causal focus and estimates of consensus: An examination of the false-consensus effect. Journal of Personality and Social Psychology, 45(3), 550-559. https://doi.org/10.1037/0022-3514.45.3.550

Heyes, C., \& Catmur, C. (2021). What Happened to Mirror Neurons? -. Perspectives on Psychological Sciences. https://doi.org/10.1177/1745691621990638

Hoffmann, F., Banzhaf, C., Kanske, P., Gärtner, M., Bermpohl, F., \& Singer, T. (2016). Empathy in depression: Egocentric and altercentric biases and the role of alexithymia. Journal of Affective Disorders, 199, 23-29. https://doi.org/10.1016/j.jad.2016.03.007

Hoffmann, F., Singer, T., \& Steinbeis, N. (2015). Children's increased emotional egocentricity compared to adults is mediated by age-related differences in conflict processing. Child Development, 86(3), 765-780. doi: 10.1111/cdev.12338

Iacoboni, M. (2009). Imitation, empathy, and mirror neurons. Annual Review of Psychology, 60, 653-670. doi: 10.1146/annurev.psych.60.110707.163604. 
Kenny, D. A., \& Acitelli, L. K. (2001). Accuracy and bias in the perception of the partner in a close relationship. Journal of Personality and Social Psychology, 80(3), 439-448. https://doi.org/10.1037/0022-3514.80.3.439

Lamm, C., Bukowski, H., \& Silani, G. (2016). From shared to distinct self-other representations in empathy: Evidence from neurotypical function and socio-cognitive disorders. Philosophical Transactions of the Royal Society B: Biological Sciences, 371(1686), 20150083.

Lamm, C., Decety, J., \& Singer, T. (2011). Meta-analytic evidence for common and distinct neural networks associated with directly experienced pain and empathy for pain. NeuroImage, 54(3), 2492-2502. https://doi.org/10.1016/j.neuroimage.2010.10.014

Lamm, C., \& Majdandžić, J. (2015). The role of shared neural activations, mirror neurons, and morality in empathy-A critical comment. Neuroscience Research, 90, 15-24. https://doi.org/10.1016/j.neures.2014.10.008

Lopez-Perez, B., \& Wilson, E. (2015). Parent-child discrepancies in the assessment of children's and adolescents' happiness. Journal of Experimental Child Psychology, 139, 249-255. https://doi.org/10.1016/j.jecp.2015.06.006

Mitchell, J. P. (2009). Inferences about mental states. Philosophical Transactions of the Royal Society B: Biological Sciences, 364(1521), 1309-1316.

Morrison, I., Löken, L. S., \& Olausson, H. (2010). The skin as a social organ | SpringerLink. Experimental Brain Reserach, 204, 305-314. doi: 10.1098/rstb.2008.0318

Murray, S. L., Holmes, J. G., Bellavia, G., Griffin, D. W., \& Dolderman, D. (2002). Kindred spirits? The benefits of egocentrism in close relationships. Journal of Personality and Social Psychology, 82(4), 563-581. https://doi.org/10.1037/0022-3514.82.4.563

Overgaauw, S., Rieffe, C., Broekhof, E., Crone, E. A., \& Güroğlu, B. (2017). Assessing Empathy across Childhood and Adolescence: Validation of the Empathy Questionnaire 
for Children and Adolescents (EmQue-CA). Frontiers in Psychology, 8, 870. https://doi.org/10.3389/fpsyg.2017.00870

Piaget, J., \& Inhelder, B. (1956). The child's conception ofspace. FJ Langdon \& JL Lunzer, trans.). London: Routledge \& Kegan Paul.

Riva, F., Lenger, M., Kronbichler, M., Lamm, C., \& Silani, G. (2022). The role of right supra-marginal gyrus and secondary somatosensory cortex in age-related differences in human emotional egocentricity. Neurobiology of Aging, 112, 102-110. https://doi.org/10.1016/j.neurobiolaging.2022.01.002

Riva, F., Triscoli, C., Lamm, C., Carnaghi, A., \& Silani, G. (2016). Emotional Egocentricity Bias Across the Life-Span. Frontiers in Aging Neuroscience, 8, 74. https://doi.org/10.3389/fnagi.2016.00074

Santiesteban, I., White, S., Cook, J., Gilbert, S. J., Heyes, C., \& Bird, G. (2012). Training social cognition: From imitation to Theory of Mind. Cognition, 122(2), 228-235. https://doi.org/10.1016/j.cognition.2011.11.004

Sebastian, C. L., Fontaine, N. M. G., Bird, G., Blakemore, S.-J., De Brito, S. A., McCrory, E. J. P., \& Viding, E. (2012). Neural processing associated with cognitive and affective Theory of Mind in adolescents and adults. Social Cognitive and Affective Neuroscience, 7(1), 53-63. https://doi.org/10.1093/scan/nsr023

Silani, G., Lamm, C., Ruff, C. C., \& Singer, T. (2013). Right Supramarginal Gyrus Is Crucial to Overcome Emotional Egocentricity Bias in Social Judgments. Journal of Neuroscience, 33(39), 15466-15476. https://doi.org/10.1523/JNEUROSCI.148813.2013

Singer, T., Seymour, B., O’doherty, J., Kaube, H., Dolan, R. J., \& Frith, C. D. (2004). Empathy for pain involves the affective but not sensory components of pain. Science, 303(5661), 1157-1162. doi: 10.1126/science.1093535. 
Southgate, V. (2020). Are infants altercentric? The other and the self in early social cognition. Psychological Review, 127(4), 505-523. https://doi.org/10.1037/rev0000182

Steinbeis, N. (2016). The role of self-other distinction in understanding others' mental and emotional states: Neurocognitive mechanisms in children and adults. Philosophical Transactions of the Royal Society B: Biological Sciences, 371(1686), 20150074. https://doi.org/10.1098/rstb.2015.0074

Theriault, J. E., Young, L., \& Barrett, L. F. (2021). The sense of should: A biologically-based framework for modeling social pressure. Physics of Life Reviews, 36, 100-136. https://doi.org/10.1016/j.plrev.2020.01.004

Trilla, I., Weigand, A., \& Dziobek, I. (2021). Affective states influence emotion perception: Evidence for emotional egocentricity. Psychological Research, 85(3), 1005-1015. https://doi.org/10.1007/s00426-020-01314-3

\section{Supplementary materials}

\section{Experimental Set-up}

Two boxes were created specifically to allow a direct view of the other participant, hiding the self, and allowing the experimenter to see and be able to touch both participants synchronously (dimensions: $40 * 17 * 20 \mathrm{~cm}$ ). Boxes were mirrored so they were respectively open on the right and left side to allow from a participant $1^{\text {st }}$ person perspective vision of the other participant's forearm only.

Participants were sitting next to each other, separated by a curtain to avoid any vision of the other, and were touched respectively on their right and left forearm (see Figure 1). Distance between the respective right and left index fingers of the two participants were kept at a constant distance of $30 \mathrm{~cm}$ (participants placed their index finger on a tape inside the box. 


\section{Supplementary Results}

Comparing biases between mothers and children groups. Comparing between bEBs and bABs in both groups across both conditions (own and other) using t-tests.

$\begin{array}{ccccc} & \boldsymbol{t} & \boldsymbol{p} & \text { Cohen's } \boldsymbol{d} & \boldsymbol{B F}^{\mathbf{1 0}} \\ \text { bEB } & -1.16 & 0.25 & -0.22 & 0.37 \\ \text { bAB } & 0.39 & 0.69 & 0.08 & 0.22\end{array}$

Comparing between EEBs, bEBs and bABs in both groups, separately for each condition (own and other) using t-tests.

\section{Own}

\begin{tabular}{ccccc}
\hline & $\boldsymbol{t}$ & $\boldsymbol{p}$ & Cohen's $\boldsymbol{d}$ & $\boldsymbol{B F}^{\mathbf{I 0}}$ \\
EBB & -1.18 & 0.24 & -0.32 & 0.48 \\
bAB & 1.19 & 0.24 & 0.32 & 0.49 \\
bEB & -0.09 & 0.93 & -0.02 & 0.28 \\
\hline & & Other & & \\
\hline EBB & $\boldsymbol{t}$ & $\boldsymbol{p}$ & Cohen's $\boldsymbol{d}$ & $\boldsymbol{B F}^{\mathbf{1 0}}$ \\
bAB & -1.41 & 0.16 & -0.38 & 0.62 \\
bEB & -0.56 & 0.57 & -0.15 & 0.31 \\
& -1.83 & 0.07 & -0.49 & 1.08
\end{tabular}

